\title{
Franck-Condon blockade in suspended carbon nanotube quantum dots
}

\author{
Renaud Leturcq ${ }^{1,2 \star}$, Christoph Stampfer ${ }^{1,3 \star}$, Kevin Inderbitzin ${ }^{1}$, Lukas Durrer ${ }^{3}$, Christofer Hierold $^{3}$, \\ Eros Mariani ${ }^{4}$, Maximilian G. Schultz ${ }^{4}$, Felix von Oppen ${ }^{4}$ and Klaus Ensslin ${ }^{1}$
}

\begin{abstract}
Understanding the influence of vibrational motion of the atoms on electronic transitions in molecules constitutes a cornerstone of quantum physics, as epitomized by the FranckCondon principle ${ }^{1,2}$ of spectroscopy. Recent advances in building molecular-electronics devices ${ }^{3}$ and nanoelectromechanical systems ${ }^{4}$ open a new arena for studying the interaction between mechanical and electronic degrees of freedom in transport at the single-molecule level. The tunnelling of electrons through molecules or suspended quantum dots ${ }^{5,6}$ has been shown to excite vibrational modes, or vibrons ${ }^{6-9}$. Beyond this effect, theory predicts that strong electron-vibron coupling strongly suppresses the current flow at low biases, a collective behaviour known as Franck-Condon blockade ${ }^{10,11}$. Here, we show measurements on quantum dots formed in suspended single-wall carbon nanotubes revealing a remarkably large electron-vibron coupling that, owing to the high quality and unprecedented tunability of our samples, allow a quantitative analysis of vibron-mediated electronic transport in the regime of strong electron-vibron coupling. This enables us to unambiguously demonstrate the Franck-Condon blockade in a suspended nanostructure. The large observed electron-vibron coupling could ultimately be a key ingredient for the detection of quantized mechanical motion ${ }^{12,13}$. It also emphasizes the unique potential for nanoelectromechanical device applications based on suspended graphene sheets and carbon nanotubes.
\end{abstract}

In a polar semiconductor, a conduction electron deforms the surrounding lattice to form a polaron state ${ }^{14}$. The formation of this quasi-particle, by combining an electron and a cloud of lattice vibrations, or phonons, strongly influences the transport properties. The possibility for localization of strongly coupled polarons was suggested by Landau more than 70 years ago $^{14}$. Recently, Koch et al. predicted that a related trapping of heavy polarons can occur in a quantum dot formed in a mechanically suspended nanostructure $\mathrm{e}^{10,11}$. In such a nanoelectromechanical system, the vibrational modes of the nanostructure can be strongly affected by the presence of electrons in the quantum dot, as they deform the embedding medium. For strong electron-phonon coupling, the deformation effectively blocks electronic transport, termed Franck-Condon blockade. By analysing electronic transport through a suspended carbon nanotube (CNT) quantum dot over a wide range of electronic states, we are able to highlight generic features of vibron-assisted electronic transport, and unambiguously confirm the Franck-Condon blockade scenario.

Scanning electron microscope images and a scheme of our suspended CNT quantum dot device are shown in Fig. 1a-c. The
CNT is electrically and mechanically connected to both source (S) and drain (D) contacts, and the central electrode acts as a suspended top-gate. A quantum dot in the CNT is formed between defects ${ }^{15}$, which are presumably created during the release process and act as local barriers. The double top- and back-gate configuration enables us to determine the location of the quantum dot below the top-gate (see Fig. 1c and Supplementary Information). Although similar data were observed on a second sample with the same geometry, only data measured on the first sample are presented here.

The exceptional quality of our sample, which is crucial for this investigation, is revealed by the well-resolved observation of multiple four-fold degeneracies of electronic states in the large-scale presentation of our data (Fig. 1d). This stems from the combined spin and valley degeneracies in clean CNTs (refs 16-18), and enables us to fully characterize the electronic properties of our device, including the electronic confinement energy $\Delta E_{\text {elec }}=6.8 \pm 2.5 \mathrm{meV}$ (see Supplementary Information).

At a finer energy scale (see Fig. 2), all of the probed Coulomb diamonds show several striking generic features. (1) Quasi-periodic lines running parallel to the edges of the Coulomb diamonds appear ubiquitously. We will see that they correspond to excited vibronic states. (2) For increasing temperatures, we observe the appearance of absorption sideband peaks within the Coulomb blockaded regions. (3) At the edge of the diamonds, there is a pronounced current suppression compared with the current magnitude for excited vibronic states. (4) Most diamonds show a significant apparent shift of their tip between positive and negative bias. (5) Finally, all of the probed diamonds show ubiquitous negative differential conductance appearing in between the excited vibronic states. We will show that most of these features are generic for transport-induced vibron excitations and are signatures of the strong electron-vibron coupling in our system.

Quasi-periodic excited states have been reported in previous experiments on suspended quantum dots. They have been interpreted as being due to the excitation of quantized phonons in the nanostructure, called vibrons ${ }^{6-9}$ : when the bias voltage matches a multiple of the energy of a vibron, $\hbar \omega_{0}$, transport is enhanced by emission of a vibron. This interpretation is confirmed in our data because the energy spacing between these excited vibronic states $\Delta E_{\mathrm{vib}}=0.8 \pm 0.2 \mathrm{meV}$ is much lower than the measured electronic level spacing. In addition, Fig. $2 \mathrm{~b}$ shows that this energy is constant over a wide range of gate voltages and does not depend on the electronic structure of the quantum dot.

The bosonic origin of the excitations is further demonstrated by the temperature dependence of the data. As temperature

\footnotetext{
${ }^{1}$ Laboratory for Solid State Physics, ETH Zurich, 8093 Zurich, Switzerland, ${ }^{2}$ Institut d'Électronique de Microélectronique et de Nanotechnologie, CNRS-UMR 8520, Department ISEN, Avenue Poincaré, BP 60069, 59652 Villeneuve d'Ascq Cedex, France, ${ }^{3}$ Micro and Nanosystems, Department of Mechanical and Process Engineering, ETH Zurich, 8092 Zurich, Switzerland, ${ }^{4}$ Institut für Theoretische Physik, Freie Universität Berlin, Arnimallee 14,14195 Berlin, Germany. ${ }^{*}$ These authors contributed equally to this work. ${ }^{\dagger}$ e-mail: renaud.leturcq@isen.iemn.univ-lille1.fr.
} 


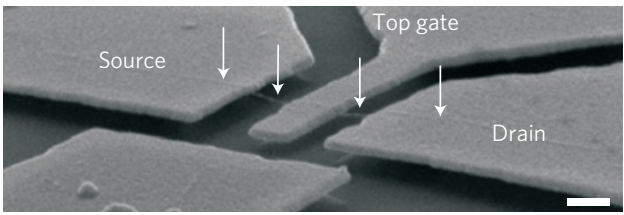

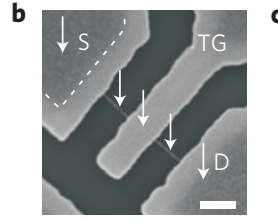

c

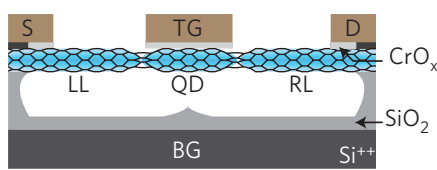

d

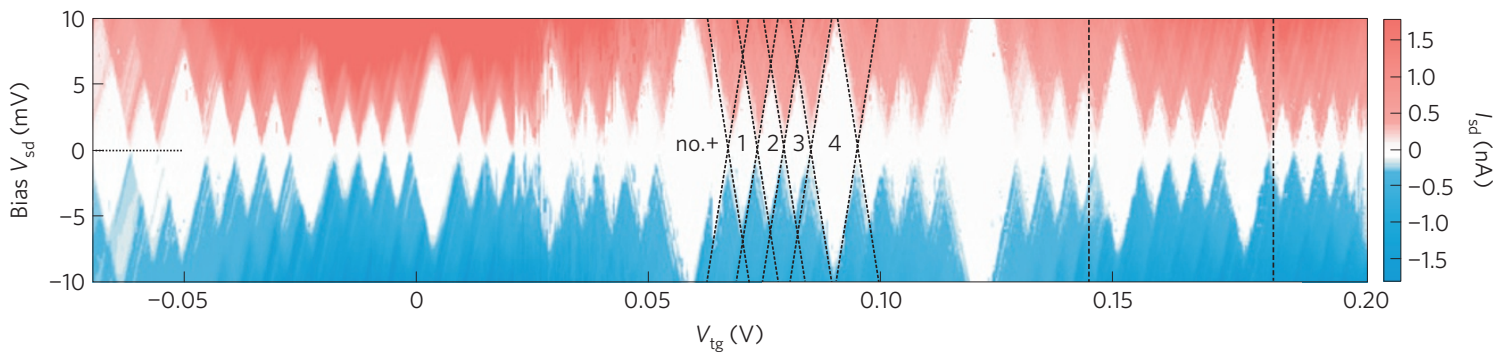

Figure 1 | Characterization of the suspended CNT quantum dot. a, Electron microscope micrograph in tilted view of the suspended CNT (white arrows) with the source (S) and drain (D) electrodes and the central top-gate (TG) electrode. $\mathbf{b}$, Top view of the device. The scale bar in $\mathbf{a}$ and $\mathbf{b}$ is $200 \mathrm{~nm}$. c, Scheme of the quantum dot (QD) device formed in the suspended CNT. As shown by the charge stability diagrams (see Supplementary Information), a quantum dot is formed under the top-gate, separated from the other parts of the nanotube by tunnel barriers. Both the suspended top-gate and the underlying back-gate (BG) can be used to tune the electronic properties of the quantum dot and the leads (LL: left lead; RL: right lead). d, Source-drain current $I_{\text {sd }}$ through the quantum dot measured as a function of the bias voltage $V_{s d}$ and the top-gate voltage $V_{\text {tg }}$, adjusting the back-gate voltage simultaneously to keep the average chemical potential in the leads constant (see Supplementary Information): $V_{\mathrm{bg}}=-0.7576 \times V_{\mathrm{tg}}+1.6784 \mathrm{~V}$. Diamond-shape regions of suppressed current are characteristic of Coulomb blockade (so-called Coulomb diamonds), and the four-fold periodicity is characteristic of quantum dots formed in clean CNTs.

is increased, we observe extra excited vibronic states appearing within the Coulomb blockade regime (Fig. 2c-f), similar to antiStokes resonances in Raman spectroscopy. This behaviour, not reported in previous experiments, is expected for excited vibronic states because higher vibrational states become populated at elevated temperature. As a consequence, electronic transport is enabled even in the Coulomb-blockade regime by absorption of a vibron. The quantitative analysis of the peak magnitude as a function of temperature confirms the bosonic origin of the absorption peak (Fig. 2g).

The very observation of vibrational sidebands is a signature of rather strong electron-vibron couplings in our CNT quantum dot. This is further highlighted by the current suppression at low bias detected in all of the probed diamonds.

Indeed, our data unambiguously confirm that this is a direct demonstration of the so-called Franck-Condon blockade ${ }^{10,11}$. When tunnelling onto the dot, the electron shifts the equilibrium coordinate of the vibron oscillator by an amount proportional to the electron-vibron coupling (Fig. 3a). The transition probability of this tunnelling process is proportional to the square of the overlap between the vibronic wavefunctions before and after tunnelling. In spectroscopy, this principle is well known as the Franck-Condon principle $e^{1,2}$ and explains the magnitude of peaks in absorption spectra. As the overlap of low-lying vibronic states is exponentially sensitive to this geometrical displacement, the ground-state-toground-state transition for vibrons is exponentially suppressed for strong electron-vibron coupling. This leads to the FranckCondon blockade of the current at low bias. At the same time, the overlap between the vibronic ground state with highly excited vibronic states, of which the wavefunctions are widely spread in space, is still significant. This enables tunnelling while exciting vibrons, as long as the bias is large enough to compensate for the vibronic energy difference, causing the observed vibrational sidebands.

This interpretation provides a quantitative understanding of the experimental data. The apparent shift of the Coulomb diamond tips for positive and negative bias observed in many cases in our experiment (Fig. 3c) and others ${ }^{5}$, is explained by the asymmetric tunnel coupling of the quantum dot with source and drain in combination with strong vibrational relaxation (so that each electronic tunnelling event starts out of the vibronic ground state). The smaller tunnel coupling determines the currentlimiting process, and energetic considerations (shown in Fig. 3e) imply that the edge of the diamond is due to ground-state-toground-state vibronic transitions for the diamond edge of one slope and due to ground-state-to-excited-state vibronic transitions for the other. As a result, the solution (see Fig. $3 \mathrm{~d}$ ) of the corresponding rate equations in Fig. 3 e reproduces the experiment in Fig. 3c quite accurately.

To extract the electron-vibron coupling $g$ despite the above asymmetry, we study the differential conductance at the gate voltage corresponding to the diamond tip (Fig. 3f,g). For equilibrated phonons, the Franck-Condon theory predicts differential conductance peaks following the progression:

$$
\left(\frac{\mathrm{d} I_{\mathrm{sd}}}{\mathrm{d} V_{\mathrm{sd}}}\right)_{n}^{\max } \propto\left|M_{0 \rightarrow n}\right|^{2} \propto \frac{\mathrm{e}^{-g} g^{n}}{n !}
$$

with $n$ being the difference in vibron quantum numbers and $\left|M_{0 \rightarrow n}\right|$ the overlap between vibronic states with 0 and $n$ vibrons. By fitting the maxima of $\mathrm{d} I_{\mathrm{sd}} / \mathrm{d} V_{\text {sd }}$ with this expression (Fig. 3f,g), we find very good agreement for most measured diamonds. Our measurement not only shows a current suppression at low bias ${ }^{5,6}$ but rather matches the whole progression for excited vibronic states, proving global consistency with the Franck-Condon picture. We deduce a large value for the electron-vibron coupling parameter $g$. For the whole set of electronic states underlying the Coulomb diamonds of Fig. 1d, the average value is $\langle g\rangle=3.3$. This is about a factor of three larger than in previous measurements ${ }^{6}$. We also observe state-to-state fluctuations of $g$ with a standard deviation of 0.9 .

To obtain a theoretical understanding of the large electron-vibron coupling constant $g$ in the quantum dot, we consider the intrinsic electron-phonon coupling of the underlying CNT. This turns out to be dominant compared with previously 
a

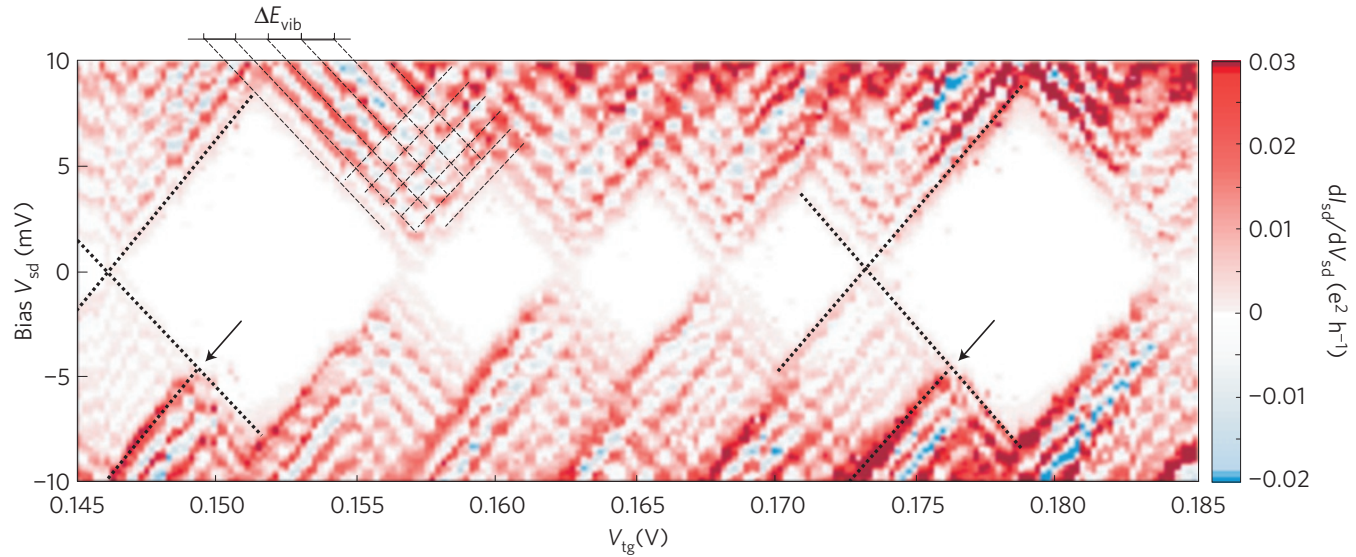

b

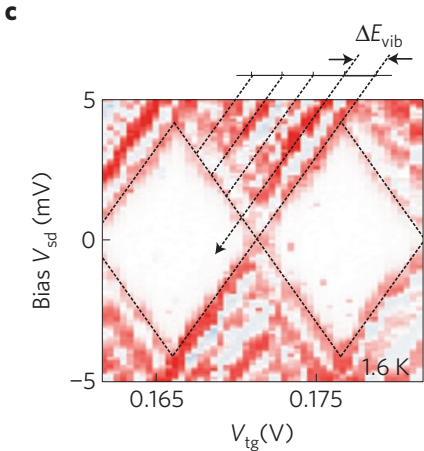

d

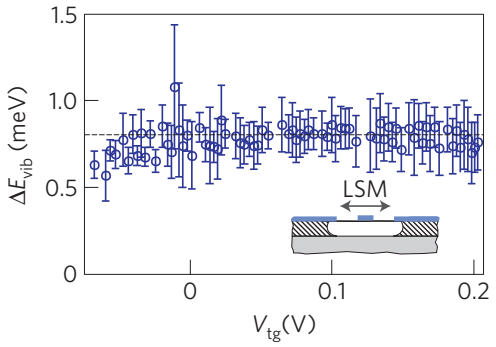

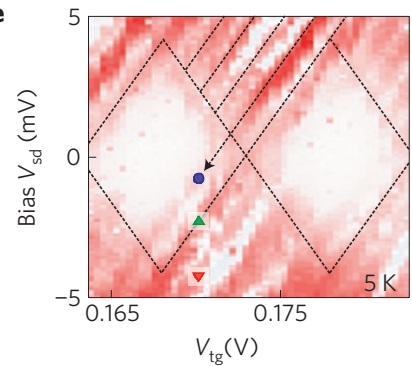

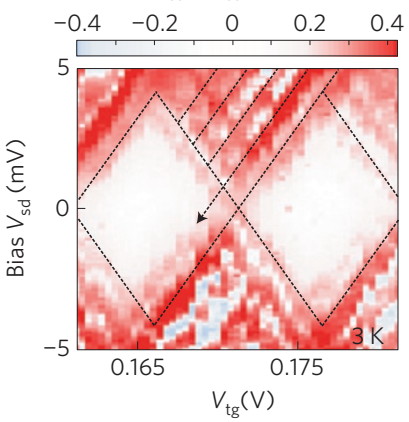

$\mathbf{f}$

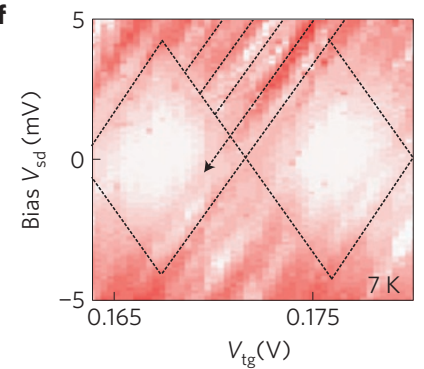

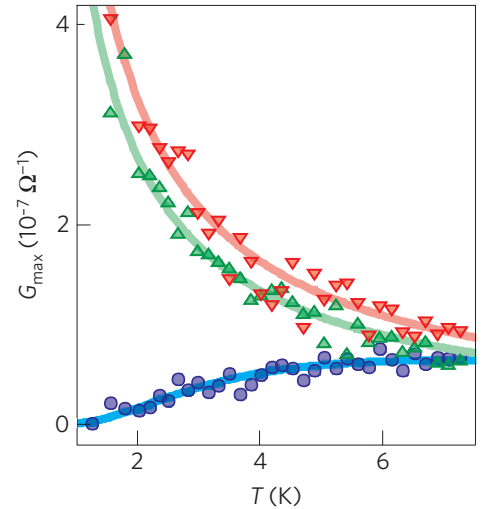

Figure 2 | Evidence and temperature dependence of vibron-assisted transport. a, Differential conductance $\mathrm{d} l_{\mathrm{sd}} / \mathrm{d} V_{\mathrm{sd}}$ for a subset of the Coulomb diamonds shown in Fig. 1d, showing the quasi-periodic excited vibronic states (see dotted lines). The arrows point to electronic excited states, visible at higher energy. b. Energy spacing of the excited vibronic states for the whole gate voltage range shown in Fig. 1d. For each point, the energy spacing is averaged over half of a Coulomb diamond, corresponding to a given electronic state, with error bars determined as the standard deviation of the mean. The horizontal dashed line represents the average value over all points, $\Delta E_{\text {vib }}=0.8 \pm 0.2 \mathrm{meV}$. This energy is compatible with the longitudinal stretching mode, for which $\Delta E_{\mathrm{vib}}=\hbar v_{\mathrm{ph}} q$, with $q=n \pi / L$ for the $n$th vibron mode in the doubly clamped nanotube and $L$ being the confinement length. Using the value of the stretching phonon group velocity in clean suspended CNTs, $v_{\mathrm{st}} \approx 2.4 \times 10^{4} \mathrm{~m} \mathrm{~s}^{-1}$ (ref. 25), we deduce the characteristic length for the first vibron mode $L \approx 65 \mathrm{~nm}$, of the same order of magnitude as the suspended parts of the nanotube and in reasonable agreement with earlier experiments ${ }^{6}$. c-f, Coulomb diamonds measured in the same region of gate voltage (same electronic state) for different temperatures, $1.6 \mathrm{~K}(\mathbf{c}), 3 \mathrm{~K}(\mathbf{d}), 5 \mathrm{~K}(\mathbf{e})$ and $7 \mathrm{~K}(\mathbf{f})$. As the temperature increases, extra conductance peaks appear in the Coulomb blockaded regions. In e, the green uptriangle and red downtriangle mark the positions of conductance peaks corresponding to tunnelling through the ground state and the first excited vibronic state (emission peak), and the blue circle to the position of a conductance peak due to the absorption of a vibron (absorption peak). $\mathbf{g}$, Maximum conductance $G_{\max }$ for tunnelling through the ground state (green uptriangle), the emission peak (red downtriangle) and the absorption peak (blue circles), corresponding to points marked in e. For the tunnelling through the ground state and the emission peak, we use as a fit $G_{\max } \propto 1 / k_{B} T$ (see green and red curves), as expected for the derivative of the Fermi distribution in the quantum Coulomb blockade regime ${ }^{26}$. For the absorption peak, we find a good fit with the product of the equilibrium population of the phonon states, given by the Bose distribution, $1 /\left(\exp \left(\hbar \omega_{0} / k_{\mathrm{B}} T\right)-1\right)$, and the derivative of the Fermi distribution, giving $G_{\max } \propto 1 / k_{\mathrm{B}} T \times 1 /$ $\left(\exp \left(\hbar \omega_{0} / k_{B} T\right)-1\right)$ (see blue curve). From the fit to the experimental data, we determine $\hbar \omega_{0}=0.96 \pm 0.08 \mathrm{meV}$, which is consistent with the energy spacing of the excited vibronic states ( $\Delta E_{\mathrm{vib}}=0.88 \pm 0.15 \mathrm{meV}$ at this value of the gate voltage).

considered extrinsic electrostatic coupling mechanisms ${ }^{6,19}$. The coupling originates from (1) the modified hopping associated with changes in the $\mathrm{C}-\mathrm{C}$ bond lengths and (2) the deformation potential due to local area variations; the deformation potential coupling constant $g_{\mathrm{D}} \approx 30 \mathrm{eV}$ is about an order of magnitude larger than the hopping-induced one $e^{20,21}$.
When combined with symmetry considerations, the existence of these two coupling mechanisms has several important consequences for the coupling strengths of the various CNT vibron modes. Longitudinal stretching modes (LSMs) and radial breathing modes couple most strongly. Their associated lattice deformations involve local area variations and produce a linear 


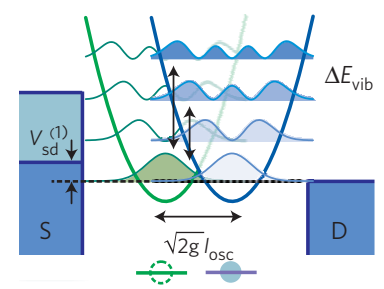

b

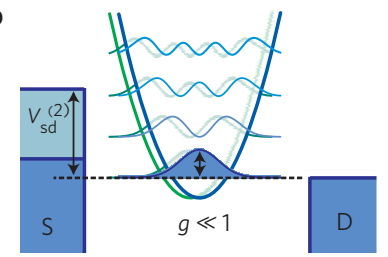

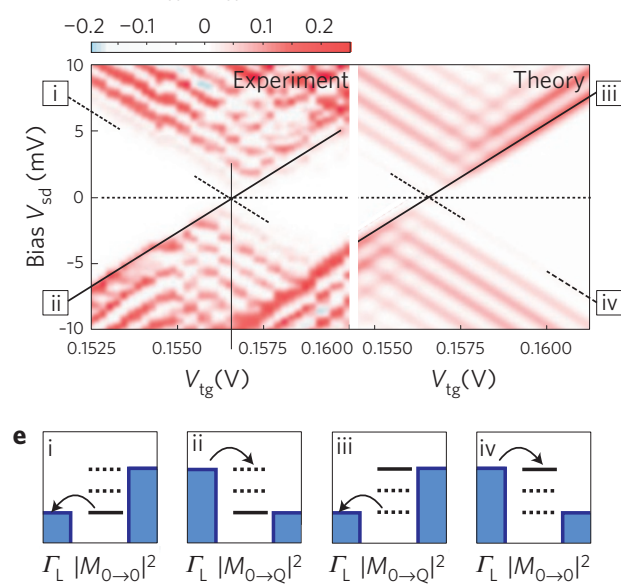

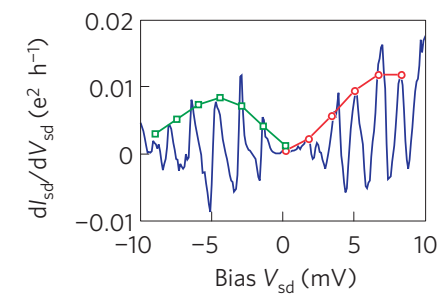

$\mathbf{g}$

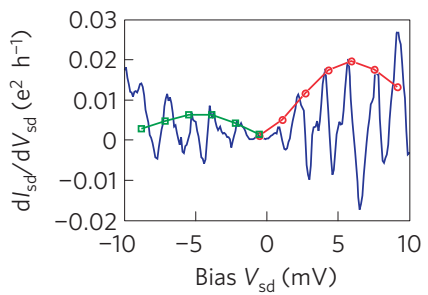

Figure 3 | Theory and analysis of Franck-Condon blockade. a,b, Schematic description of the displacement of the vibron potential for strong ( $g \gg 1$ ) (a) and weak $(g \ll 1)(\mathbf{b})$ electron-vibron coupling $g$, with $N$ (green curve) and $N+1$ electrons (blue curve) in the dot. The shift for $g \gg 1$ (a) suppresses the transitions between low-lying vibronic states and thus the current in the low-bias regime $\left(V_{\text {sd }}^{1}\right)$, causing the Franck-Condon blockade. In parallel, it opens transitions to excited vibronic states in the high-bias regime $\left(V_{\text {sd }}^{2}\right.$, see vertical double-headed arrows), yielding vibrational sidebands. In contrast, for $g \ll 1$ the shift is essentially absent, enabling ground-state-to-ground-state vibronic transitions (see vertical double-headed arrow). At the same time, the vibronic ground state before tunnelling is essentially orthogonal to all excited vibronic states after tunnelling. This suppresses the transition probabilities to highly excited vibronic states and hence the vibrational sidebands. c, Zoom into a part of the Coulomb diamonds (around $V_{\mathrm{tg}}=0.1570 \mathrm{~V}$ ) showing the suppression of the low-bias transport and the apparent shift of the Coulomb diamond tip at positive bias with respect to that at a negative one. $\mathbf{d}$, Simulated Coulomb diamonds in the same conditions as in the experiment (with $k_{\mathrm{B}} T=0.15 \hbar \omega_{0}, \Gamma_{\mathrm{R}}=10 \Gamma_{\mathrm{L}}, g=3.3$ ) for strongly asymmetric tunnel coupling between the leads and the dot, $\Gamma_{\mathrm{R}} \gg \Gamma_{\mathrm{L}}$. e, Schematic diagrams of the rate-limiting processes through the opaque left barrier for the extremal lines (dotted in c and $\mathbf{d}$ ) of the Coulomb diamond. For the extremal line with negative slope, the relevant process implies tunnelling through the left barrier with no vibronic excitation ( $i$ and iv). In the presence of strong vibronic relaxation, each electronic tunnelling event starts out of the vibronic ground state. Thus, these processes lead to a current proportional to $\Gamma_{\mathrm{L}}\left|M_{0 \rightarrow 0}\right|^{2}$ involving the Franck-Condon overlap $M_{0 \rightarrow 0}$ between vibronic ground states, and the Franck-Condon suppression of vibronic transitions manifests itself also at large bias. The current at the extremal line with positive slope is also predominantly limited by tunnelling through the left junction, but as $V_{\text {sd }}$ increases inelastic excitation of vibrons becomes energetically allowed (ii and iii). The strong vibronic relaxation implies that these processes are associated with vibronic transitions involving $M_{0} \rightarrow Q, w h e r e Q=\operatorname{lnt}\left[e V_{s d} / \hbar \omega_{0}\right]$ is the maximum allowed vibronic quantum number (as long as $M_{0 \rightarrow Q}$ increases with Q). Because for strong electron-vibron coupling $M_{0 \rightarrow Q} \gg M_{0 \rightarrow 0}$, the resulting differential conductance peak proportional to $\Gamma_{\mathrm{L}}\left|M_{0 \rightarrow Q}\right|^{2}$ increases with the bias voltage. f, Differential conductance measured for $V_{\mathrm{tg}}=0.0678 \mathrm{~V}$. Representative fits of the maxima with the Franck-Condon progression (equation (1)) enable us to extract the parameters $g=3.5$ (negative bias voltage, green squares) and $g=5.0$ (positive bias voltage, red circles). Peaks that deviate strongly from the progression at large bias voltage are due to electronic excited states. $\mathbf{g}$, Same as in $\mathbf{f}$, for $V_{\operatorname{tg}}=0.1570 \mathrm{~V}$, giving $g=3.0$ (negative bias voltage, green squares) and $g=4.5$ (positive bias voltage, red circles).

shift of the electronic energies, resulting in a linear deformation potential interaction. Twist modes also couple linearly, but they are area preserving and couple only through the weaker hopping-induced mechanism. Finally, bending modes have only a weak quadratic interaction because, for symmetry reasons, the electronic energy does not depend on the sign of the associated deformation of the CNT.

We can thus identify the relevant vibron mode probed in our data. Indeed, of the strongly coupled vibrons, only the LSM is in a frequency range compatible with our experimental observations (see Fig. 2b), as discussed previously ${ }^{6}$. The relevant dimensionless coupling constant $g$ for the occurrence of the Franck-Condon blockade is given by (the square of) the shift of the equilibrium coordinate measured in units of the amplitude (oscillator length) of the vibronic quantum fluctuations. We compute this quantity in the framework of the effective Dirac Hamiltonian for the low-energy electronic properties of CNTs, combined with the theory of elasticity for the description of the vibron mode (see details in Supplementary Information). Using literature values for the elastic constants of graphene $e^{21}$, this calculation yields a maximal coupling of

$$
g_{\max }^{(\mathrm{LSM})} \simeq \frac{10}{L_{\perp}[\mathrm{nm}]}
$$

for the lowest LSM. An important consequence of our analysis is that the coupling constant is strongly sample dependent. It is inversely proportional to the CNT circumference $L_{\perp}$, and proportional to the electronic matrix element of the deformation potential. The matrix element vanishes for the lowest longitudinal stretching mode when electrons and vibrons are confined to the same region of the CNT. It becomes maximal when the electronic wavefunction is sharply localized around a region of maximum strain, yielding the estimate in equation (2).

Atomic force microscope measurements indicate that our CNTs typically have a circumference of a few nanometres. Thus, our value for $g$ given in equation (2) is consistent with the observation of the Franck-Condon blockade, which requires $g>1$. At the same time, the coupling constants extracted from experiment are slightly larger than our estimate. Here, the defects creating the electronic confinement could have a major role: first they help confine the wavefunction in a region of maximum strain; second they could also modify the vibrons and/or the deformation potential. Other observations also indicate that experiments exhibit more pronounced vibronic effects in transport than theory suggests: vibrational sidebands are accompanied by negative differential conductance, as already reported ${ }^{6}$, whereas theory predicts a step-like $I-V$ characteristic. In addition, the experiment shows vibrational absorption sidebands within the 
Coulomb blockade diamonds, which are absent in theoretical simulations. We have checked that these intriguing enhancements are not related to asymmetric coupling of the dot to the leads (see Supplementary Information). The underlying reasons are not understood at present.

\section{Methods}

Devices were fabricated from highly doped silicon wafer substrates covered by $200 \mathrm{~nm}$ silicon oxide. Single-walled CNTs (SWNTs) were grown randomly on the oxide substrate by chemical vapour deposition based on dispersed ferritin catalysts ${ }^{22}$. Electron beam lithography was used to pattern metallic electrodes and gate structures $(2 / 30 \mathrm{~nm} \mathrm{Cr} / \mathrm{Au}$ ) around (selected) individual SWNTs (Fig. 1b). Finally, diluted HF ( $4 \%$ for $5 \mathrm{~min}$ ) etching followed by critical point drying completes the device fabrication (Fig. 1c). It is crucial that the $\mathrm{Cr}$ layer oxidizes after the etching and drying processes, as well as when exposed to environmental air, to form the top-gate oxide. For a more detailed process description, see refs 23 , 24. Measurements were carried out in a variable temperature ${ }^{4} \mathrm{He}$ cryostat at a base temperature of $T=1.3 \mathrm{~K}$. We have measured the two-terminal conductance through the SWNT device by applying a symmetric d.c. bias voltage $V_{\text {sd }}$ while measuring the current through the quantum dot device with a resolution better than $10 \mathrm{fA}$. For differential conductance measurements, a small a.c. bias, $V_{a c}=48 \mu \mathrm{V}$ was superimposed on $V_{\mathrm{sd}}$ and the differential conductance was measured with lock-in techniques. Measurements have been done in two different devices, with quantum dots formed at different locations along the CNT. All configurations show similar results as the one presented here.

Received 3 December 2008; accepted 23 February 2009; published online 6 April 2009

\section{References}

1. Franck, J. Elementary processes of photochemical reactions. Trans. Farad. Soc. 21, 536-542 (1926).

2. Condon, E. A theory of intensity distribution in band systems. Phys. Rev. 28, 1182-1201 (1926).

3. Joachim, C., Gimzewski, J. K. \& Aviram, A. Electronics using hybrid-molecular and mono-molecular devices. Nature 408, 541-548 (2000).

4. Ekinci, K. L. \& Roukes, M. L. Nanoelectromechanical systems. Rev. Sci. Instr. 76, 061101 (2005).

5. Weig, E. M. et al. Single-electron-phonon interaction in a suspended quantum dot phonon cavity. Phys. Rev. Lett. 92, 046804 (2004).

6. Sapmaz, S., Jarillo-Herrero, P., Blanter, Y. M., Dekker, C. \& van der Zant, H. S. J. Tunneling in suspended carbon nanotubes assisted by longitudinal phonons. Phys. Rev. Lett. 96, 026801 (2006).

7. Park, H. et al. Nanomechanical oscillations in a single- $\mathrm{C}_{6} 0$ transistor. Nature 407, 57-60 (2000).

8. Yu, L. H. et al. Inelastic electron tunneling via molecular vibrations in single-molecule transistors. Phys. Rev. Lett. 93, 266802 (2004).

9. Pasupathy, A. N. et al. Vibration-assisted electron tunneling in $\mathrm{C}_{140}$ transistors. Nano Lett. 5, 203-207 (2005).

10. Koch, J. \& von Oppen, F. Franck-Condon blockade and giant Fano factors in transport through single molecules. Phys. Rev. Lett. 94, 206804 (2005).

11. Koch, J., von Oppen, F. \& Andreev, A.V. Theory of the Franck-Condon blockade regime. Phys. Rev. B 74, 205438 (2006)
12. Knobel, R. G. \& Cleland, A. N. Nanometre-scale displacement sensing using a single electron transistor. Nature 424, 291-293 (2003).

13. LaHaye, M. D., Buu, O., Camarota, B. \& Schwab, K. C. Approaching the quantum limit of a nanomechanical resonator. Science 304, 74-77 (2004).

14. Landau, L. D. Electron motion in crystal lattice. Phys. Z. Sowjet. 3, 664-665 (1933).

15. Postma, H. W. C., Teepen, T., Yao, Z., Grifoni, M. \& Dekker, C. Carbon nanotube single-electron transistors at room temperature. Science $\mathbf{2 9 3}$ 76-79 (2001)

16. Liang, W., Bockrath, M. \& Park, H. Shell filling and exchange coupling in metallic single-walled carbon nanotubes. Phys. Rev. Lett. 88, 126801 (2002).

17. Buitelaar, M. R., Bachtold, A., Nussbaumer, T., Iqbal, M. \& Schönenberger, C. Multiwall carbon nanotubes as quantum dots. Phys. Rev. Lett. 88, 156801 (2002)

18. Sapmaz, S. et al. Electronic excitation spectrum of metallic carbon nanotubes. Phys. Rev. B 71, 153402 (2005).

19. Braig, S. \& Flensberg, K. Vibrational sidebands and dissipative tunneling in molecular transistors. Phys. Rev. B 68, 205324 (2003).

20. Woods, L. M. \& Mahan, G. D. Electron-phonon effects in graphene and armchair $(10,10)$ single-wall carbon nanotube. Phys. Rev. B 61 10651-10663 (2000)

21. Suzuura, H. \& Ando, T. Phonons and electron-phonon scattering in carbon nanotubes. Phys. Rev. B 65, 235412 (2002)

22. Durrer, L. et al. SWNT growth by CVD on Ferritin-based iron catalyst nanoparticles towards CNT sensors. Actuators Sensors Actuators B 132 , 485-490 (2008)

23. Stampfer, C. et al. Nano-electromechanical displacement sensing based on single-walled carbon nanotubes. Nano Lett. 6, 1449-1453 (2006).

24. Stampfer, C., Jungen, A. \& Hierold, C. Fabrication of discrete nanoscaled force sensors based on single-walled carbon nanotubes. IEEE Sensors J. 6, 613-617 (2006).

25. Dresselhaus, M. S. \& Eklund, P. C. Phonons in carbon nanotubes. Adv. Phys. 49, 705-814 (2000).

26. Kouwenhoven, L. P. et al. in Mesoscopic Electron Transport (eds Sohn, L. L. Kouwenhoven, L. P. \& Schön, G.) 105-214 (NATO ASI Ser. E 345, Kluwer, 1997)

\section{Acknowledgements}

We thank K. Kobayashi, L. Wirtz, D. Obergfell, M. Muoth, M. L. Roukes and S. Ilani for helpful discussions. This work was supported by the TH-18/03-1 Grant and the Swiss National Science Foundation through NCCR Nanoscience, as well as Sfb658 and SPP 1243. Sample fabrication was carried out at the ETH FIRST and CLA Laboratories.

\section{Author contributions}

C.S., R.L. and K.E. planned the experiment. C.S., L.D. and C.H. fabricated the samples. R.L., K.I. and C.S. carried out the measurements and analysed the results. E.M., M.G.S and F.v.O. did the theoretical analysis and carried out the simulation. R.L., C.S. and E.M. wrote the paper, with inputs from M.G.S., F.v.O. and K.E.

\section{Additional information}

Supplementary information accompanies this paper on www.nature.com/naturephysics. Reprints and permissions information is available online at http://npg.nature.com/ reprintsandpermissions. Correspondence and requests for materials should be addressed to R.L. 